\section{Device for Opening and Closing Magenta Vessels for Micropropagation}

\author{
Loren D. Gautz and \\ Charles W. Wong
}

Additional index words. tissue culture, repetitive work injury

Summary. A device is described that can be used to open and close Magenta vessels used for micropropagation. Performance of the device is reported and compared favorably to unassisted manual opening and closing of vessels. Benefits include elimination of a potentially physically damaging (e.g., carpal tunnel syndrome) manual operation.

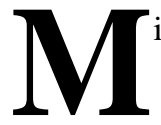
icropropagation is the invitro culture of small (micro) cuttings for propagation of plants. The cuttings are induced to produce multiple new shoots through manipulation of the chemical and physical environment. By this process, plant material of the same genotype can be reproduced rapidly and can be kept free of viral, bacterial, and fungal diseases. Because of these benefits, micropropagation has been adopted widely. More then 250 laboratories in the United States are engaged in micropropagation of many types of plants. Five to 10 of these laboratories produce more than 5 million plants annually (Wochock, 1987). Many of these laboratories use the Magenta GA-7 vessel.

In Hawaii, the Hawaiian Sugar Planters' Assn. (HSPA) was using micropropagation to supply sugar planters with micropropagated sugarcane transplants at rates as high as

Agricultural Engineering, University of Hawaii at Manoa, Honolulu, HI 96822.

Hawaii Inst. of Tropical Agriculture and Human Resources Journal Series no. 3790. Mention of brand names is for clarity only and is not an endorsement. Funding was made available for this research by the Office of the Vice President for Research and Graduate Studies, Univ. of Hawaii at Manoa, Honolulu.
100,000 plants/week. Current practice is to use five generations for multiplication, including the rooting phase. Each generation takes 14 days and gives a shoot multiplication of four times. A vessel must be opened at the beginning, at the middle to change the growing medium, and at the end of each generation. This means that, for sustained production, 21,826 vessels were opened per week with a crew of six people. Workers have complained of pain similar to the onset of carpal tunnel syndrome from repetitive opening of vessels. In a study to determine the optimal conditions for micropropagation of sugarcane, Walker et al. (1991) determined that the liquid growing medium should be changed twice during the 14-day growing cycle. Using two medium changes instead of one would increase the number of vessels that must be opened per week by $25 \%$.

Similar to many other micropropagation laboratories, HSPA uses the Magenta GA-7 vessel. Several factors lead to the selection of this vessel and are outside the scope of this study.

Other than many recommendations to automate the handling of vessels used for micropropagation, no references were found relating to the opening of vessels. More than 800 U.S. patents related to openers were found in the U.S. Patent Office Gazette. These consist of openers for everything from bags, bottles, and cans, to oysters and wooden boxes. There are 54 patents for opening containers. The U.S. Patent Office does not have a classification for plastic container or plastic box. Of the patented openers, none were found that related to opening a friction-fit lid on a plastic box such as the GA-7 vessel used for micropropagation.

\section{Basic data}

The GA-7 vessel is made from polycarbonate plastic and is nearly cubical in shape; nominally $80 \mathrm{~mm}$ on a side. The lid is a polyethylene material that has a friction-fit to the vessel. When the lid is closed completely, a gap between the lid and the vessel $\approx 1$ $\mathrm{mm}$ wide and $1 \mathrm{~mm}$ deep extends around the outside of the vessel at the interface. Although the lips on the vessel and the lid make accidental contact with the interior of the vessel or lid during opening by hand highly unlikely, the force exerted during the opening of some vessels causes occasional mishaps such as dropping the lid or spilling the contents of the vessel.

The force required to pull a lid straight off from a vessel was determined using an Instron Universal Testing machine. The vessel was held down by a fixture gripping two opposite sides. The lid was fastened to the crosshead using a gripper with grasping jaws in the lid-vessel gap on either two opposing sides or two opposing corners.

Tests were run with four vessels and four lids chosen at random from the current stock. The sample included both old and new vessels and tops. All 16 possible combinations of the vessels and lids were tested at two crossheadspeeds: 0.4 and $10.6 \mathrm{~mm} \cdot \mathrm{s}^{-1}$. The maximum, minimum, and mean peak force attained during opening the 16 combinations is shown in Table 1. Because only 16 tests were made, the force used for engineering design calculations should be taken as at least the maximum force experienced for the type of gripper used.

The force using the opposing side grip was independent of pull rate. Because the force using the opposing corner grip was not independent of pull rate and greater than when using the opposing side grip, opposing corner gripping was not considered for development of an opening device.

\section{Description of device}

The device is a hand-held tool to assist in opening friction-fit containers (Fig. 1). The prototype was designed to open Magenta GA-7 containers, but can be modified easily to open other containers. The device consists of a handles and two grippers; one stationary and the other movable. The handle is a pistol grip shape with a knob at the top to aid in control while not gripping a vessel. The grippers are made of strips of $0.9-\mathrm{mm}$ (20 USStd. ga.) stainless steel sheet metal that is bent at the ends at right angles to form a small jaw to engage the lid at the gap between it and the vessel. The stationary gripper is attached to the bottom of the handle and grabs one side of the lid. The movable gripper is a simple cantilevered beam mounted to the top of the handle. A jaw at the free (lower) end of the movable gripper grabs the far side of the lid. The operator holds the device with the stationary gripper adjacent to the heel of the hand and 
Table 1. Peak forcer $(N)$ to open vessels at two pull rates $\left(\mathbf{m m} \cdot \mathrm{s}^{-1}\right)$.

\begin{tabular}{llcccccc}
\hline Grasp & Pull rate & Trials & Maximum & Minimum & Mean & Population (SD) & 95 percentile \\
\hline Side-side & 0.4 & 16 & 41 & 4 & 24.8 & 13.6 & 36 \\
& 10.6 & 16 & 38 & 4 & 24.2 & 11.9 & 37 \\
& Avg. & 32 & 41 & 4 & 24.5 & 12.6 & 37 \\
Corner- & & & & & & & \\
corner & 0.4 & 16 & 44 & 5 & 24.6 & 12.3 & 39 \\
& 10.6 & 16 & 58 & 7 & 35.4 & 17.0 & 54 \\
Combined & Avg. & 32 & 58 & 5 & 30.0 & 15.6 & 54 \\
& 0.4 & 32 & 44 & 4 & 24.7 & 12.8 & \\
& 10.6 & 32 & 58 & 4 & 29.8 & 15.5 & \\
\hline
\end{tabular}

the movable gripper under the fingers. The shape of the handle makes it possible to hold the device with the thumb and palm without applying pressure to the movable gripper with the fingers.

After clasping the vessel with one hand, the operator places the device
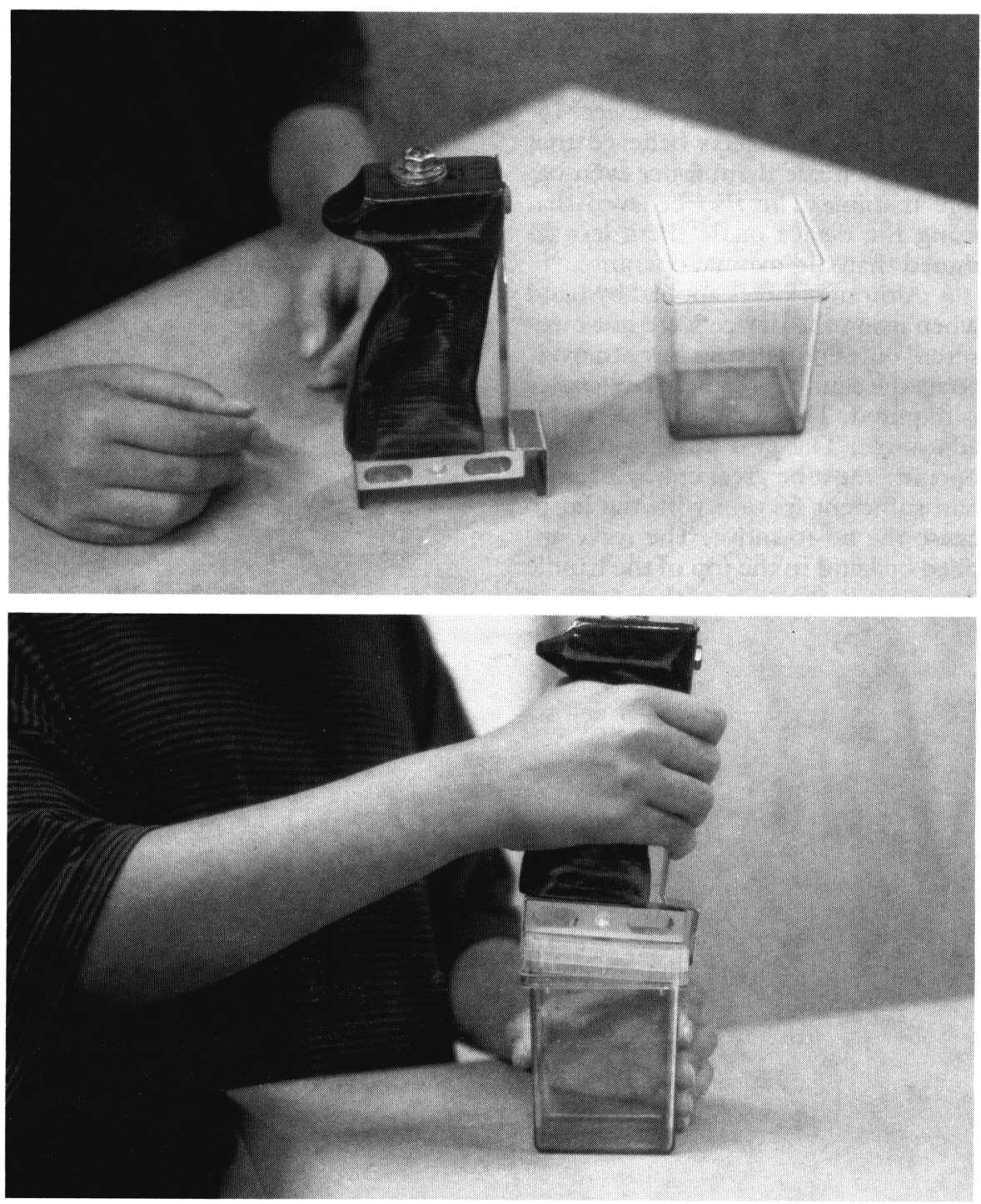

Fig. 1. Device for opening and closing Magenta GA-7 vessels (top) andproper initial application to opening (bottom). with the base of the thumb, causing the device to rotate slightly and the near side of the lid to slide up. The operator then lifts the lid off with a little force. Depending on the laboratory practice, the lid and device can be placed aside by setting them down on the lip of the lid or rotating the device forward to hold the lid in a vertical position. The lid may remain in the device. Suspending the device on some sort of hanger is not recommended. The lid cannot remain securely in the device, and no savings in motion is obtained.

The lid can be replaced using the device. Because the friction-fit is sufficiently tight to hold the vessel, the closed vessel can be placed aside with the device while the other hand acquires another vessel. This gives a balanced motion to the hands, necessary for optimal working efficiency. With training and properworkspace arrangement, placing the device aside is unnecessary except when this hand is needed for pouring media, or some other peripheral task.

\section{Evaluation}

The device was evaluated by timing only the lid removal and hand movement to the next vessel to be opened. For each trial, 11 vessels were placed in an arc at an easy reach from the operator. Timing started at the completion of opening the first vessel and ended. with the completion of opening the last vessel. Therefore, 10 vessels were opened during the timed interval. The timer was accurate to $0.01 \mathrm{~s}$. More than 10 trials were made with each subject using the device, and a similar number of trials with each subject opening vessels manually. Some trials were not used for the average because of various avoidable errors in the trial. A complete test session for a give subject took $>50 \mathrm{~min}$ and $>200$ vessels were opened.

Six subjects volunteered for the time study. Subjects 1-4 were male and subjects 5 and 6 were female. Subject 1 was trained with the device, but had very little experience with the hand-opening ofvessels. Subject 2 was trained with the device and was experienced with both the device and handopening. Subject 3 was a total novice, with no training or experience prior to the trials. Subjects 4-6 had opened many vessels by hand in a research laboratory, but had never used the 
Table 2. Time (s) to open 10 vessels.

\begin{tabular}{|c|c|c|c|c|c|c|c|c|c|}
\hline \multirow[b]{3}{*}{ Subject } & \multicolumn{3}{|c|}{ Means } & \multicolumn{3}{|c|}{ SB of means } & \multicolumn{3}{|c|}{ df } \\
\hline & \multirow[b]{2}{*}{ Hand } & \multicolumn{2}{|c|}{ Device } & \multirow[b]{2}{*}{ Fand } & \multicolumn{2}{|c|}{ Device } & \multirow[b]{2}{*}{ Hand } & \multicolumn{2}{|c|}{ Device } \\
\hline & & Novice & Trained & & Novice & Trained & & Novice & Trained \\
\hline 1 & 22.4 & & $19.8 * *$ & 0.23 & & 0.46 & 9 & & 12 \\
\hline 2 & 18.5 & & $15.1^{* *}$ & 0.68 & & 0.36 & 9 & & 9 \\
\hline 3 & 19.9 & 22.5 & $* *$ & 0.51 & 0.49 & & 9 & 13 & \\
\hline 4 & 15.1 & 16.0 & 14.8 & 0.62 & 0.58 & 0.39 & 11 & 12 & 12 \\
\hline 5 & 20.1 & 15.2 & $18.6^{*}$ & 0.61 & 0.43 & 0.43 & 12 & 12 & 12 \\
\hline 6 & 23.3 & 25.5 & $21.4^{*}$ & 0.76 & 0.67 & 0.66 & 12 & 12 & 12 \\
\hline
\end{tabular}

device before. The last three subjects were first tested without training with the device. Following initial trials, they were given instruction and supervised practice using the device and then were tested with the device again. Instruction and practice did not exceed $15 \mathrm{~min}$. Hand and device trials were interspersed randomly. Unfortunately, once trained, the subject cannot be tested as untrained, making randomization of trained and untrained conditions impossible.

Mean times for a subject to open vessels by hand and with the device (Table 2) were compared statistically using Student's $t$ test. All four subjects who had not been trained with the device had slower times with the device compared to manual opening. For only one of these four (subject 4) was the difference not highly significant statistically (99\% confidence). Three of these subjects were given training, bringing the total number of trained subjects to five. All subjects trained with the device had lower opening times compared to hand-opening. Only one subject (subject 4) did not exhibit a statistically significant difference. Even though this is a small sample, the probability for all subjects to lower their times purely by random effects would be similar to getting five heads in five tosses with a fair coin (i.e., $3 \%$ ). This indicates that some training is required even with this simple device. Also, with training, there is a time advantage when using the device. Interestingly, all subjects believed that the device made them faster even before training. The also believed that using the device made them less fatigued than did manual opening.

Although forces applied by hand when using the device were not measured, only enough grip force to overcome the cantilevered beam resistance is required. This force is $<1 \mathrm{~N}$ and is adjustable. The grip force in manual opening must be great enough to obtain sufficient friction with the lid to cause the lid to move. The force applied by hand to the top of the handle is less than pulling force applied during manual opening, because of the lever action of the handle. The hand holding the vessel experiences the same forces with either method, but the uncertain forces observed during manual opening are not experienced using the device.

\section{Conclusion}

The device described here can be used to make the opening of Magenta GA-7 vessels, used commonly for micropropagation, less fatiguing. There is no time penalty for using the device. In fact, there is good probability that some time will be saved with the device. Because the gripping force that must be applied is substantially less than without, and because the wrist takes a more natural position during opening of the vessels, there is much less chance for repetitive stress injuries that are presently the cause for complaint by workers. Also, when using the device, the vessel remains stable and the lid does not suddenly pop out of tightly gripped fingers as it is wrenched off the vessel by hand.

\section{Literature Cited}

Walker, P.N., J.P. Harris, and L.D. Gautz. 1991. Optimal environment for sugarcane micropropagation. Trans. of ASAE 34: 2609-2614.

Wochock, Z.S. 1987. Status of crop improvement through tissue culture. Comb. Proc. Intl. Plant Prop. Soc. 36:72-77. 